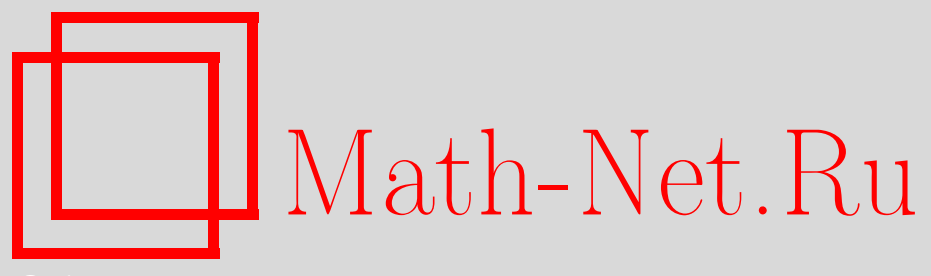

Н. К. Макина, Э. А. Назирова, Я. Т. Султанаев, О методах исследования асимптотического поведения решений сингулярных дифференциальных уравнений, $M a-$ тем. заметки, 2014, том 96, выпуск 4, 627-632

DOI: https://doi.org/10.4213/mzm10531

Использование Общероссийского математического портала Math-Net.Ru подразумевает, что вы прочитали и согласны с пользовательским соглашением http://www . mathnet.ru/rus/agreement

Параметры загрузки:

IP : 54.162 .27 .143

26 апреля 2023 г., 10:30:05

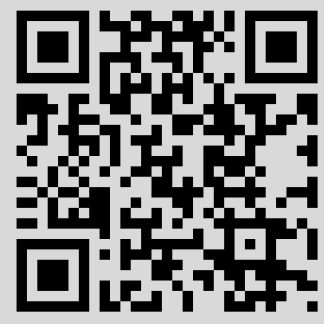




\section{О методах исследования асимптотического поведения решений сингулярных дифференциальных уравнений}

\section{Н. К. Макина, Э. А. Назирова, Я. Т. Султанаев}

1. Известно [1], [2], [3], что для решений уравнения

$$
y^{\prime \prime}+q(x) y=0, \quad 0<x<\infty,
$$

где $q(x)$ - вещественная, положительная и такая, что $q(x) \rightarrow+\infty$ при $x \rightarrow \infty$, справедливы ВКБ асимптотические формулы:

$$
y_{1,2} \sim \frac{1}{\sqrt[4]{q(x)}} \exp \left\{ \pm i \int_{x_{0}}^{x} \sqrt{q(t)} d t\right\}
$$

где $q(x)$ удовлетворяет следующим условиям:

1) $q^{\prime}(x), q^{\prime \prime}(x)$ при достаточно большом $x_{0}$ не меняют знак в интервале $\left[x_{0},+\infty\right)$;

2) $q^{\prime}(x)=o\left(q^{\alpha}(x)\right), 0<\alpha<3 / 2$.

При этом в [1], [2] использовался метод Левинсона замены уравнения (1) системой уравнений первого порядка и приведением ее к $L$-диагональному виду, а в [3] использовалась замена Лиувилля. Первый метод более громоздкий, зато в отличии от второго может быть применен к уравнениям порядка выше второго.

$L$-диагональные системы - это системы вида

$$
u^{\prime}(x)=\Lambda(x) u(x)+\Theta(x) u(x),
$$

где $\Theta(x)$ - суммируемая на $\left[x_{0}, \infty\right)$ матрица. Заметим, что условия 1$)$ и 2$)$ довольно жесткие, но они обеспечивают суммируемость матрицы $\Theta(x)$.

Целью настоящей заметки является задача о существенном расширении класса потенциалов $q(x)$, к которым можно применить метод Левинсона.

2. Рассмотрим следующее уравнение:

$$
-y^{\prime \prime}-q(x) y-\left(q_{1}(x)-q(x)\right) y=0 .
$$

Обозначим $u(x)=q_{1}(x)-q(x)$. Здесь $q(x)-$ функция, удовлетворяющая условиям 1$\left.), 2\right)$ п. 1. Перейдем от данного уравнения к системе, введя в рассмотрение вектор-столбец $Y=$ $\left(y, y^{\prime}\right)$ :

$$
\begin{aligned}
Y^{\prime} & =A(x) Y \\
A(x)=A_{1}(x)+A_{2}(x), & A_{1}(x)=\left(\begin{array}{cc}
0 & 1 \\
-q(x) & 0
\end{array}\right), \quad A_{2}(x)=\left(\begin{array}{cc}
0 & 0 \\
-u(x) & 0
\end{array}\right) .
\end{aligned}
$$

Найдем собственные значения матрицы $A_{1}(x)$, обозначая через $I$ тождественную матрицу размера $2 \times 2$ :

$$
\operatorname{det}\left(A_{1}-\omega \cdot I\right)=0, \quad \omega^{2}+q(x)=0, \quad \omega_{1,2}(x)= \pm i q^{1 / 2}(x)
$$

Пусть матрица $T(x)$ приводит $A_{1}$ к диагональному виду:

$$
T^{-1}(x) A_{1}(x) T(x)=\Lambda(x), \quad \Lambda(x)=\operatorname{diag}\left\{\omega_{1}(x),-\omega_{1}(x)\right\} .
$$

Работа второго автора выполнена при поддержке Российского фонда фундаментальных исследований (грант № 12-01-00567-а); работа третьего автора выполнена при поддержке Министерства образования и науки РФ в рамках госзадания вузам РФ в сфере научной деятельности.

DOI: $10.4213 / \mathrm{mzm} 10531$ 
Известно, что элементы матрицы $T(x)$ определяются с точностью до умножения элементов каждого столбца на скалярную функцию, при этом эти функции могут быть выбраны так, чтобы выполнялось соотношение

$$
\left(T^{-1}(x) T^{\prime}(x)\right)_{i i}=0, \quad i=1,2 .
$$

Тогда $T(x)$ имеет вид

$$
\begin{gathered}
T(x)=\delta(x)\left(\begin{array}{cc}
1 & 1 \\
\omega_{1}(x) & -\omega_{1}(x)
\end{array}\right), \quad \delta(x)=q^{-1 / 4}(x) \\
T^{-1}(x)=\frac{1}{2}\left(\begin{array}{cc}
\frac{1}{\delta} & \frac{\delta}{i} \\
\frac{1}{\delta} & -\frac{\delta}{i}
\end{array}\right), \quad T^{-1}(x) T^{\prime}(x)=: C(x)=\frac{\delta^{\prime}}{\delta}\left(\begin{array}{cc}
0 & 1 \\
1 & 0
\end{array}\right) .
\end{gathered}
$$

Сделав в системе (2) замену

$$
Y=T \cdot U
$$

придем к системе

$$
U^{\prime}=\Lambda(x) U-C(x) U+T^{-1}(x) A_{2}(x) T(x) .
$$

Пусть теперь матрица $G(x)$ такова, что $G(x) \Lambda(x)-\Lambda(x) G(x)=-C(x)$,

$$
G(x)=\frac{\delta^{\prime}(x) \delta(x)}{2 i}\left(\begin{array}{cc}
0 & -1 \\
1 & 0
\end{array}\right) .
$$

Сделаем в (4) замену

$$
U=(I+G(x)) W
$$

тогда получим систему

$$
W^{\prime}=\Lambda W-(I+G)^{-1} C G W-(I+G)^{-1} G^{\prime} W-(I+G)^{-1} T^{-1} A_{2} T(I+G) W .
$$

Введем в рассмотрение функции

$$
a(x)=\frac{\delta^{\prime}(x) \delta(x)}{2 i}, \quad \Delta(x)=1+a^{2}(x), \quad p(x)=u(x) \cdot \delta^{2}(x) .
$$

Пусть для краткости

$$
D:=(I+G)^{-1} C G+(I+G)^{-1} G^{\prime}, \quad p(x) \cdot B:=(I+G)^{-1} T^{-1} A_{2} T(I+G) .
$$

Несложно выписать элементы матриц $D, B$, с учетом обозначений (7):

$$
\begin{aligned}
D(x) & =\frac{\delta^{\prime}(x)}{2 i \Delta(x)}\left(\begin{array}{cc}
1 & a(x) \\
-a(x) & 1
\end{array}\right)+\frac{a^{\prime}(x)}{\Delta(x)}\left(\begin{array}{cc}
a(x) & -1 \\
1 & a(x)
\end{array}\right) \\
& =\frac{\delta^{\prime}(x) / 2 i+a^{\prime}(x)}{\Delta(x)}\left(\begin{array}{cc}
1+a(x) & a(x)-1 \\
1-a(x) & 1+a(x)
\end{array}\right)
\end{aligned}
$$

И

$$
B(x)=\left(\begin{array}{cc}
1-a^{2}(x) & (a(x)-1)^{2} \\
-(a(x)+1)^{2} & a^{2}(x)-1
\end{array}\right) \rightarrow I, \quad x \rightarrow \infty .
$$

Полагая в системе (6)

$$
\left(\begin{array}{l}
w_{1} \\
w_{2}
\end{array}\right)=e^{\int \omega_{1}(t) d t}\left(\begin{array}{l}
z_{1} \\
z_{2}
\end{array}\right)
$$

получаем систему уравнений

$$
\begin{aligned}
& z_{1}^{\prime}=-\left(d_{11}(x)+p(x) \cdot b_{11}(x)\right) z_{1}-\left(d_{12}(x)+p(x) \cdot b_{12}(x)\right) z_{2}, \\
& z_{2}^{\prime}=-2 \cdot \omega_{1}(x) z_{2}-\left(d_{21}(x)+p(x) \cdot b_{21}(x)\right) z_{1}-\left(d_{22}(x)+p(x) \cdot b_{22}(x)\right) z_{2} .
\end{aligned}
$$


Предполагая, что система (11) имеет решение, удовлетворяющее условиям

$$
\begin{aligned}
& \lim _{x \rightarrow \infty} z_{1}(x)=1, \\
& \lim _{x \rightarrow \infty} z_{2}(x)=0,
\end{aligned}
$$

и поступая аналогично доказательству леммы 1 в [1; с. 288], перейдем от системы (11) к системе интегральных уравнений

$$
\begin{aligned}
& z_{1}=1-\int_{x}^{\infty}\left[\left(d_{11}(t)+p(t) \cdot b_{11}(t)\right) z_{1}(t)-\left(d_{12}(t)+p(t) \cdot b_{12}(t)\right) z_{2}(t)\right] d t \\
& z_{2}=-\int_{x}^{\infty} \psi(x, t)\left[\left(d_{21}(t)+p(t) \cdot b_{21}(t)\right) z_{1}(t)-\left(d_{22}(t)+p(t) \cdot b_{22}(t)\right) z_{2}(t)\right] d t
\end{aligned}
$$

где

$$
\psi(x, t)=\exp \left\{2 \int_{x}^{t} \omega_{1}(s) d s\right\} .
$$

Интегрируя по частям слагаемые в правых частях уравнений, содержащие функцию $p(x)$, после преобразований можем привести (12) к следующему виду:

$$
\begin{aligned}
\left(\begin{array}{l}
z_{1} \\
z_{2}
\end{array}\right)=\left(\begin{array}{l}
1 \\
0
\end{array}\right)+P(x) \cdot\left(\begin{array}{ll}
b_{11} & b_{12} \\
b_{21} & b_{22}
\end{array}\right)\left(\begin{array}{l}
z_{1} \\
z_{2}
\end{array}\right) \\
+\int_{x}^{\infty} P(t)\left(\begin{array}{ll}
\alpha_{11} & \alpha_{12} \\
\alpha_{21} & \alpha_{22}
\end{array}\right)\left(\begin{array}{l}
z_{1} \\
z_{2}
\end{array}\right) d t-\int_{x}^{\infty}\left(\begin{array}{ll}
d_{11} & d_{12} \\
d_{21} & d_{22}
\end{array}\right)\left(\begin{array}{l}
z_{1} \\
z_{2}
\end{array}\right) d t
\end{aligned}
$$

где

$$
P(x)=\int_{x}^{\infty} p(t) d t=\int_{x}^{\infty} \frac{q_{1}(t)-q(t)}{\sqrt{q(t)}} d t
$$

и

$$
\begin{aligned}
& \alpha_{11}=b_{11}^{\prime}-b_{11} d_{11}-p b_{11}^{2}-b_{12}\left(d_{21}+b_{21} p\right), \\
& \alpha_{12}=b_{12}^{\prime}-b_{11}\left(d_{12}+p b_{12}\right)-b_{12}\left(2 \omega_{1}+d_{22}+b_{22} p\right), \\
& \alpha_{21}=\left(\psi b_{21}\right)^{\prime}-\psi b_{21}\left(d_{11}+p b_{11}\right)-\psi b_{22}\left(d_{21}+b_{21} p\right), \\
& \alpha_{22}=\left(\psi b_{22}\right)^{\prime}-\psi b_{21}\left(d_{12}+p b_{12}\right)-\psi b_{22}\left(2 \omega_{1}+d_{22}+b_{22} p\right) .
\end{aligned}
$$

Потребуем выполнения условия

$$
\left|P(x) \cdot \omega_{1}\right| \leqslant r(x), \quad x>x_{0}
$$

где $r(x) \in L(0, \infty)$.

Перепишем систему (13) в операторной форме. Введем в рассмотрение $M$ - множество всех непрерывных на $\left[x_{0}, \infty\right)$ функций с конечным пределом при $x \rightarrow+\infty$, а также множество $M \times M=\widetilde{M}$.

Введем на множестве $M$ норму

$$
\|\phi\|=\sup _{\left[x_{0}, \infty\right)}|\phi(x)| .
$$

На множестве $\widetilde{M}$ также определим норму по правилу

$$
\|x\|=\max \left\{\left\|x_{1}\right\|,\left\|x_{2}\right\|\right\} .
$$

Тогда норма оператора $A[x]$ есть

$$
\|A\|=\sup _{x \in \widetilde{M}} \frac{\|A[x]\|}{\|x\|} .
$$


Заметим, что если $A$ - оператор умножения на матрицу, то согласованная норма будет иметь вид

$$
\|A\|=\max _{j=1,2}\left\{\left\|a_{1 j}\right\|+\left\|a_{2 j}\right\|\right\} .
$$

Введем в рассмотрение оператор

$$
\begin{gathered}
K[z]=P(x) \cdot B z+\int_{x}^{\infty} P(t) \Upsilon(t) z d t-\int_{x}^{\infty} D(t) z d t, \\
z=\left(\begin{array}{c}
z_{1} \\
z_{2}
\end{array}\right), \quad(\Upsilon(t))_{i j}=\alpha_{i j}(t) .
\end{gathered}
$$

Можно показать, что при выполнении условий (1), (2) из п. 1 и условия (14) оператор $K$ действует из множества $\widetilde{M} \rightarrow \widetilde{M}$. Тогда система (13) примет вид

$$
z=e_{1}+K[z], \quad(I-K)[z]=e_{1}, \quad z=(I-K)^{-1}\left[e_{1}\right],
$$

где $e_{1}$ - единичный двумерный вектор.

Если $\|K\|<1$, то существует решение уравнения (15), причем такое, что

$$
\lim _{x \rightarrow \infty} z=e_{1}
$$

Покажем, что $\|K\|<1$. Для этого вычислим действие оператора $K[z]=: f$. С учетом (8), (9)

$$
\begin{aligned}
\left|f_{1}\right| \leqslant \mid & P(x) \cdot\left(b_{11} z_{1}+b_{12} z_{2}\right)|+| \int_{x}^{\infty} P(t)\left(\alpha_{11} z_{1}+\alpha_{12} z_{2}\right) d t|+| \int_{x}^{\infty}\left(d_{11} z_{1}+d_{12} z_{2}\right) d t \mid \\
\leqslant \mid & P(x) \mid\left(\left|b_{11}\right|\left|z_{1}\right|+\left|b_{12}\right| z_{2} \mid\right) \\
\quad & \quad+\int_{x}^{\infty}\left(\left|P(t) \alpha_{11}\right|\left|z_{1}\right|+\left|P(t) \alpha_{12}\right|\left|z_{2}\right|\right) d t+\int_{x}^{\infty}\left(\left|d_{11}\right|\left|z_{1}\right|+\left|d_{12}\right|\left|z_{2}\right|\right) d t \\
\leqslant \mid & P(x) \mid\left(\left|b_{11}\right||| z_{1}\left\|+\left|b_{12}\right|\right\| z_{2} \|\right) \\
& \quad+\int_{x}^{\infty}\left(\left|P(t) \alpha_{11}\right|\left\|z_{1}\right\|+\left|P(t) \alpha_{12}\right|\left\|z_{2}\right\|\right) d t+\int_{x}^{\infty}\left(\left|d_{11}\right|\left\|z_{1}\right\|+\left|d_{12}\right|\left\|z_{2}\right\|\right) d t \\
\leqslant \mid & P(x)||\left|B\||||z|+\| z\left\|\int_{x}^{\infty}\left(\left|P(t) \alpha_{11}\right|+\left|P(t) \alpha_{12}\right|\right) d t+\right\| z \| \int_{x}^{\infty}\left(\left|d_{11}\right|+\left|d_{12}\right|\right) d t,\right.
\end{aligned}
$$

и

$$
\left\|f_{1}\right\| \leqslant\|z\|\left(|P(x)|\|B\|+\int_{x}^{\infty}\left(\left|P(t) \alpha_{11}\right|+\left|P(t) \alpha_{12}\right|\right) d t+\int_{x}^{\infty}\left(\left|d_{11}\right|+\left|d_{12}\right|\right) d t,\right) .
$$

При выполнении условия (14) очевидно, что

$$
\lim _{x \rightarrow \infty} P(x)=0, \quad \lim _{x \rightarrow \infty} p(x)=0 .
$$

Заметим, что функция $a(x) \in L(0, \infty)\left(a(x)=\delta^{\prime}(x) \delta(x), \delta(x) \rightarrow 0, x \rightarrow \infty\right)$; следовательно,

$$
\lim _{x \rightarrow \infty} a(x)=0 .
$$

Учитывая это, получим, что $\|B(x)\|=: B_{0}=$ const; тогда $\|P(x) \cdot B(x)\|<\varepsilon$ при $x>x_{0}$ для достаточно большого $x_{0}$.

Учитывая (7) и то, что $\delta^{\prime}(x)=-1 / 4 q^{-5 / 4} \cdot q^{\prime}(x)(x) \in L(0, \infty)$, при выполнении условий (1), (2) из п. 1 верно также

$$
\int_{x_{0}}^{\infty}\left|\delta^{\prime}(x)\right| d x<\infty
$$


И

$$
\begin{aligned}
a(x) & =\frac{\delta^{\prime}(x) \delta(x)}{2 i}=\frac{1}{4 i}\left(\delta^{2}(x)\right)^{\prime}=\frac{1}{4 i}\left(q^{-1 / 2}(x)\right)^{\prime}, \\
a^{\prime}(x) & =\frac{1}{4 i}\left(q^{-1 / 2}(x)\right)^{\prime \prime}=-\frac{1}{8 i}\left(q^{-3 / 2}(x) q^{\prime}(x)\right)^{\prime}=-\frac{1}{8 i}\left(-\frac{3}{2} q^{-5 / 2}(x) q^{\prime 2}(x)+q^{-3 / 2}(x) q^{\prime \prime}(x)\right) \\
& =-\frac{1}{8 i} q^{-3 / 2}(x)\left(-\frac{3}{2} q^{-1}(x) q^{\prime 2}(x)+q^{\prime \prime}(x)\right) .
\end{aligned}
$$

В силу условий (1), (2) из п. 1

$$
\int_{x_{0}}^{\infty}\left|d_{i j}(x)\right| d x<\infty .
$$

Из условий (7) и (14) также следует

$$
\begin{array}{cc}
\lim _{x \rightarrow \infty}\left|\alpha_{11}(x)\right|=0, & \lim _{x \rightarrow \infty}\left|\alpha_{21}(x)\right|=0, \\
\alpha_{12} \sim \text { const } \cdot q^{1 / 2}(x), & \alpha_{22} \sim \text { const } \cdot q^{1 / 2}(x),
\end{array}
$$

откуда

$$
\left|P(x) \alpha_{12}(x)\right| \leqslant \text { const } \cdot r(x), \quad\left|P(x) \alpha_{22}(x)\right| \leqslant \text { const } \cdot r(x) .
$$

Учитывая последнюю оценку и (16), для $x>x_{0}$ при достаточно больших $x_{0}$ имеем

$$
\begin{gathered}
|P(x)|+\int_{x}^{\infty}\left(\left|P(t) \alpha_{11}\right|+\left|P(t) \alpha_{12}\right|\right) d t+\int_{x}^{\infty}\left(\left|d_{11}\right|+\left|d_{12}\right|\right) d t<\varepsilon, \\
\left\|f_{1}\right\|_{M} \leqslant \varepsilon\|z\|_{\widetilde{M}} .
\end{gathered}
$$

Аналогично можно получить оценки

$$
\left\|f_{2}\right\|_{M} \leqslant \varepsilon\|z\|_{\widetilde{M}}, \quad\|K[z]\| \leqslant \varepsilon\|z\|, \quad\|K\| \leqslant \varepsilon<1 .
$$

Таким образом, мы показали, что система (15) имеет решение такое, что

$$
\lim _{x \rightarrow \infty} z=e_{1} .
$$

Возвращаясь с помощью обратных замен (3), (5), (10), приходим к следующему:

$$
\begin{aligned}
& Y=T U=T(I+G) W=T(I+G) e^{\int \omega_{1}(x) d x}\left(\begin{array}{l}
z_{1} \\
z_{2}
\end{array}\right), \\
& Y=\delta(x)\left(\begin{array}{cc}
1 & 1 \\
i / \delta^{2}(x) & -i / \delta^{2}(x)
\end{array}\right)\left(\left(\begin{array}{cc}
1 & 0 \\
0 & 1
\end{array}\right)+a(x)\left(\begin{array}{cc}
0 & -1 \\
1 & 0
\end{array}\right)\right) e^{\int \omega_{1}(x) d x}\left(\begin{array}{l}
1 \\
0
\end{array}\right)(1+o(1)), \\
& Y=e^{i \int q^{1 / 2}(x) d x}\left(\left(\begin{array}{c}
q^{-1 / 4} \\
i q^{1 / 4}
\end{array}\right)+a(x)\left(\begin{array}{c}
q^{-1 / 4} \\
i q^{1 / 4}
\end{array}\right)\right)(1+o(1)) .
\end{aligned}
$$

Таким образом, нами была доказана следующая теорема.

Теорема. Пусть для функиии $q(x)$ выполнены условия (1), (2) из n. 1, а так же условие (14). Тогда уравнение $-y^{\prime \prime}-q(x) y-\left(q_{1}(x)-q(x)\right) y=0$ имеет ФСР $\left\{y_{1}(x), y_{2}(x)\right\}$ такую, что при $x \rightarrow \infty$

$$
\begin{aligned}
& y_{1}(x)=q^{-1 / 4} e^{i \int q^{1 / 2}(x) d x}(1+o(1)), \\
& y_{1}^{\prime}(x)=i q^{1 / 4} e^{i \int q^{1 / 2}(x) d x}(1+o(1)), \\
& y_{2}(x)=q^{-1 / 4} e^{-i \int q^{1 / 2}(x) d x}(1+o(1)) . \\
& y_{2}^{\prime}(x)=-i q^{1 / 4} e^{-i \int q^{1 / 2}(x) d x}(1+o(1)) .
\end{aligned}
$$


3. Примеры. Введем в рассмотрение функцию

$$
P_{1}(x)=\int_{x}^{\infty} u(x) d x .
$$

Пусть $P_{1}(x)$ удовлетворяет условию

$$
\left|P_{1}(x)\right|=\left|\int_{x}^{\infty} u(x) d x\right| \leqslant r_{1}(x),
$$

где $r_{1}(x)$ - монотонная положительная функция и $r_{1}(x) \in L(0, \infty)$. Аналогично [4] можно доказать оценку

$$
\left|P(x) q^{1 / 2}\right|<r(x)
$$

Нетрудно показать, что при выполнении условия (17) условие (14) так же выполняется.

ПримеР 1. Функция $u(x)=q_{1}(x)-q(x)=c(1+x)^{\alpha} \sin (1+x)^{\beta}, \beta>\alpha+2, \alpha>0$, а функция $q(x)$ удовлетворяет всем условиям $(1),(2)$.

Пример 2. Функция $u(x)=q_{1}(x)-q(x)=c e^{\alpha x} \sin e^{\beta x}, \beta>\alpha>0$, а функция $q(x)$ удовлетворяет всем условиям (1), (2).

ПримеР 3. Функция $q_{1}(x)=x^{\alpha} \sin ^{2} x^{\beta}, \beta>\alpha+2, \alpha>0$, а функция $q(x)$ удовлетворяет всем условиям (1), (2).

\section{СПИСОК ЦИТИРОВАННОЙ ЛИТЕРАТУРЫ}

[1] М.А. Наймарк, Линейнъе дифференииальные операторь, Наука, М., 1969. [2] М.В. Федорюк, Асимптотические методы для линейных обыкновенных дифбереницальных уравнений, Справочная математическая библиотека, Наука, М., 1983. [3] Ф. Олвер, Введение в асимптотические методы и специальные функиии, Наука, М., 1978. [4] Х. Х. Муртазин, Я. Т. Султанаев, Матем. заметки, 28:4 (1980), 545-553. [5] Н. Ф. Валеев, Я. Т. Султанаев, Матем. заметки, 65:6 (1999), 932-938. [6] Я. Т. Султанаев, Докл. АН СССР, 284:3 (1985), 551-555. [7] Л. К. Кусаинова, А. Ж. Монашова, А. А. Шкаликов, Матем. заметки, 93:4 (2013), 630-633.

\section{Н. К. Макина}

Евразийский национальный университет

им. Л.Н.Гумилева, Казахстан

E-mail: nasgul28@mail.ru

\section{Э. А. Назирова}

Башкирский государственный университет, г. Уфа

E-mail: ellkid@gmail.com

\section{Я. Т. Султанаев}

Башкирский государственный педагогический

университет им. М. Акмуллы, г. Уфа

E-mail: sultanaevyt@gmail.com
Поступило 20.04.2014 